\title{
Aktuelle Diagnostik der ambulant erworbenen Pneumonie
}

\author{
Diagnosis of Community-Acquired Pneumonia
}

B. Schaaf, J. Rupp

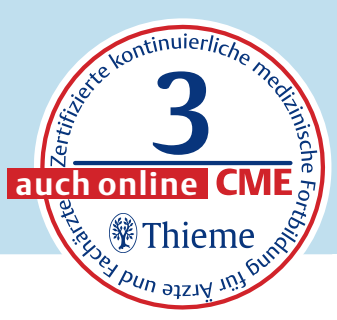

\section{Zusammenfassung}

Eine definitive Diagnose einer ambulant erworbenen Pneumonie ist entscheidend, um rechtzeitig die entsprechende Therapie und Versorgung einzuleiten. Häufiger dient die Diagnostik aber dem Ausschluss einer Pneumonie, um unnötige Antibiotikagaben zu vermeiden. Goldstandard in der Diagnosestellung ist in Zusammenhang mit anamnestischen und klinischen Befunden weiterhin die Röntgenaufnahme der Thoraxorgane. Moderne Entzündungsmarker wie das C-reaktive Protein und in Zukunft möglicherweise das Prokalzitonin helfen in der Differenzialdiagnose und Verlaufsuntersuchung. Eine mikrobiologische Erregerdiagnostik wird bei ambulant behandelten Patienten in der Regel nicht empfohlen. Bei hospitalisierten Patienten hat neben der klassischen Kultur der Urinantigen-Test auf Legionellen einen besonderen Stellenwert. Andere mikrobiologische Verfahren sind hingegen nur für Einzelfälle und Epidemien sinnvoll.

\section{Einleitung}

Pneumonien sind Entzündungen des Lungenparenchyms, die durch Mikroorganismen verursacht werden. Um die Diagnose einer ambulant erworbenen Pneumonie (community aquired pneumonia $=$ CAP) $z u$ stellen und eine adäquate Therapie zu verordnen, stehen dem Arzt neben klinischen Befunden, bildgebende Verfahren und eine breite Labordiagnostik mit Entzündungswerten sowie mikrobiologische Untersuchungen zur Verfügung. Zusätzlich können bei differenzialdiagnostischen Problemen eventuell weitere Untersuchungen notwendig sein. Welche Diagnostik im Einzelnen eingesetzt wird, steht im Ermessen des behandelnden Arztes. Eine ausgedehnte Diagnostik kann zwar zusätzliche Informationen geben, ob diese jedoch in jedem Fall prognostisch oder ökonomisch gerechtfertigt ist, ist Gegenstand der aktuellen Diskussion. Aus pneumologisch-infektiologischer Sicht sollen durch die Diagnostik zwei Ziele erreicht werden:

Durch Ausschluss einer Pneumonie und z.B.

Nachweis einer unkomplizierten akuten

Bronchitis können unnötige Antibiotikagaben vermieden werden

Eine Risiko-adaptierte Therapie verbessert möglicherweise die Prognose.

\section{Diagnosestellung}

Anamnese, klinische Untersuchung, RöntgenThoraxaufnahme, Entzündungswerte und Erregernachweis führen zur Diagnose Pneumonie.

\section{Klinische Befunde}

Symptomatik. Eine sichere klinische Diagnose einer Pneumonie ist anhand der Symptome nicht möglich. Typisch sind bei immunkompetenten Erwachsenen allgemeines Krankheitsgefühl, Husten, Sputum, verstärkter Atemarbeit, Fieber oder Hypothermie. Diese Symptome können auch bei Infektionen des oberen Respirationstrakts, bei akuter und chronischer Bronchitis und nicht infektiösen Erkrankungen (z. B. Herzinsuffizienz, Lungenarterienembolie, Vaskulitis, Malignom, Atelektase) vorkommen. Selbst beim Vorhandensein mehrerer Symptome liegt die Wahrscheinlichkeit einer CAP nur bei ca. 50\% [1,2] ( Tab. 1).

Körperliche Untersuchung. Häufige Befunde sind Dyspnoe mit erhöhter Atemfrequenz und eine Tachykardie, ggf. mit arterieller Hypotonie. Perkutorisch findet sich bei ausgedehnten Infiltrationen und/oder parapneumonischem Pleuraerguss ein abgeschwächter Klopfschall und in der Auskultation können ohrnahe Rasselgeräusche und Bronchialatmen vorliegen. Die Befunde der körperlichen Untersuchung sind wie die Symptome unspezifisch. Besondere Beachtung verdient die Tatsache, dass sich die klinische Präsentation der CAP mit steigendem Alter verändert. Im höheren Alter werden oligosymptomatische Verläufe mit extrapulmonalen Symptomen wie zunehmende
Bibliografie

Dol $10.1055 / \mathrm{s}-2006-932223$

Pneumologie 2006; 60; 547-558

(c) Georg Thieme Verlag KG

Stuttgart · New York

ISSN 0934-8387

Korrespondenzadresse

Bernhard Schaaf

Medizinische Klinik III Universitätsklinikum Schleswig-Holstein (UKSH), Campus Lübeck

Ratzeburger Allee 160 23538 Lübeck

schaaf@uni-luebeck.de 
Tab. 1 Symptomatik und Befunde bei Patienten mit Pneumonie (adaptiert nach Höffken [1]): Weder die Symptomatik noch die Befunde sind spezifisch genug, um eine Pneumonie zu diagnostizieren

\begin{tabular}{|l|l|}
\hline Symptomatik & Untersuchungsbefund \\
\hline allgemeines Krankheitsgefühl & Inspektion: Dyspnoe und Tachypnoe \\
\hline Fieber oder Hypothermie & Palpation: Tachykardie, Ggf. arterielle Hypotonie \\
\hline Husten & Perkussion: abgeschwächter Klopfschall \\
\hline eitriger Auswurf & Auskultation: \\
& fein- bis mittelblasige klingende ohrnahe Rasselgeräusche \\
& Bronchialatmen \\
\hline Dyspnoe & Schwäche, Hinfälligkeit \\
\hline „Grippale“ Symptome wie Myalgien, Arthralgien, Zephalgien \\
\hline
\end{tabular}

Schwäche oder Hinfälligkeit beobachtet [2] ( Tab. 1).

in der Differenzialdiagnose (z. B. Tuberkulose, Tumoren, Lungenarterienembolie, Herzinsuffizienz, Tab. 1 )

in der Prognoseabschätzung (multilobäre Infiltrate oder großer/beidseitiger Pleuraergüsse $=$ schlechtere Prognose) [5].

Röntgen-Thoraxaufnahme. Aufgrund der fehlenden Diagnosesicherheit durch die Anamnese und Untersuchung wird bei allen Patienten mit Verdacht auf CAP eine Röntgen-Thoraxaufnahme empfohlen, auch wenn in der ambulanten Praxis die zeitnahe Durchführung und Befundung im Einzelfall schwierig sein kann [1]. Eine posteroanteriore und eine laterale Röntgen-Thoraxaufnahme gilt als Goldstandard für die Diagnosestellung eines pneumonischen Infiltrates, wenn gleichzeitig klinische und/oder mikrobiologische Daten die Diagnose unterstützen. Der Nachweis eines Infiltrats kann vor allem bei leichtgradiger CAP mit nur geringer Infiltratausbildung allerdings schwierig sein [3]. Zudem kann die Röntgen-Thoraxaufnahme initial, insbesondere bei seltenen Pneumonien wie z. B. der PneumozystisInfektion, noch normal aussehen. Die RöntgenThoraxaufnahme differenziert zudem nicht ausreichend zwischen unterschiedlichen Erregern der CAP [4]

Zur Diagnose einer CAP ist auch im ambulanten Bereich eine Röntgen-Thoraxaufnahme für die Abgrenzung zur akuten Bronchitis wichtig, da bei akuter Bronchitis Antibiotika nicht indiziert sind. Eine Kontrolle der RöntgenThoraxaufnahme nach abgeheilter Pneumonie ist bei Patienten mit persistierenden Symptomen oder bei Verdacht auf Bronchialkarzinom erforderlich.

Der wesentliche klinische Nutzen der RöntgenThoraxaufnahme liegt:

in der Abgrenzung zur akuten Bronchitis im Nachweis von Komplikationen (Abszess, Pneumothorax, Pleuraerguss/Empyem) chiale Biopsie beweist ein Alveolarzellkarzinom.
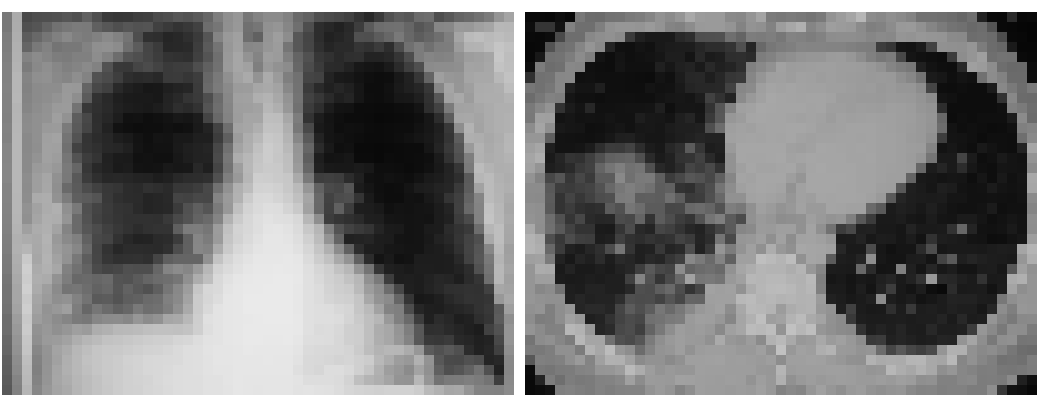

Eine Kontrolle der Röntgen-Thoraxaufnahme nach abgeSymptomen oder bei Verdacht auf Bronchialkarzinom erforderlich.

Computertomographie der Thoraxorgane. Die Computertomographie der Thoraxorgane ist generell sensitiver als die Röntgen-Thoraxaufnahme im Nachweis von interstitiellen Infiltraten, Einschmelzungen, Lymphknotenvergrößerungen und Empyemen. Einige Patienten haben typische klinische Zeichen einer CAP, jedoch radiologisch kein Infiltrat. Eine Dünnschicht-Computertomographie (High-Resolution-Computertomographie, HRCT) kann in diesen Fällen häufig ein Infiltrat zeigen, die klinische Relevanz ist jedoch nicht geprüft [7]. In der Differenzialdiagnose ist die Computertomographie der Thoraxorgane in der Abgrenzung eines Tumors und einer Lungenarterienembolie sehr wertvoll. Bei immundefizienten Patienten kann die Computertomographie unter anderem hinweisend sein für eine invasive Aspergillose.

Die Computertomographie der Thoraxorgane ist bei immunkompetenten Patienten in den meisten Fällen nicht notwendig. Sie wird hauptsächlich bei Komplikationen, Therapieversagen, in der Differenzialdiagnostik und bei heilter Pneumonie ist bei Patienten mit persistierenden immundefizienten Patienten eingesetzt. 


\section{Die CRP-Werte sind bei bakterieller CAP erhöht und nur in Einzelfällen normal, aber:}

CRP-Erhöhung ist nicht spezifisch für CAP

niedrige CRP-Werte $(<10-20 \mathrm{mg} / \mathrm{l})$ machen eine CAP unwahrscheinlich

hohe CRP-Werte ( $>100 \mathrm{mg} / \mathrm{l}$ ) machen eine CAP wahrscheinlich

Legionellen und Pneumokokkenpneumonien haben höhere CRP-Werte

- fehlender Abfall des CRP-Wertes innerhalb von 4 Tagen auf 50 \% spricht für Therapieversagen

\section{Klinisch-chemische Diagnostik}

Blutbild. Das Blutbild ist typischerweise Bestandteil der Routineblutdiagnostik der stationär behandelten CAP-Patienten. Eine Leukozytose von über $15000 / \mathrm{ml}$ macht eine bakterielle Pneumonie wahrscheinlich, ein normales Blutbild schließt eine Pneumonie jedoch nicht aus. Zusätzlich wurden in mehreren Studien sowohl eine Leukopenie $<4000 / \mathrm{ml}$ als auch eine Leukozytose $>20000 / \mathrm{ml}$ bei Aufnahme mit einer erhöhten Letalität assoziiert [8].

Bei hospitalisierten Patienten gehört das Blutbild zusammen mit den Elektrolyten, den Nierenwerten und den Leberwerten zur Routineblutdiagnostik. Bei ambulanten Patienten ist die Untersuchung des Blutbildes im Einzelfall zu erwägen.

C-reaktives Protein. Das CRP wurde initial als Pneumokokken-bindendes Protein, welches die Complement-Aktivierung triggert, entdeckt. Verschiedene Studien zeigen, dass ein niedriges CRP, das heißt Werte unter $10-20 \mathrm{mg} / \mathrm{l}$ eine Pneumonie mit einer Sensitivität und Spezifität von ca. 95\% unwahrscheinlich machen [9]. Für die Praxis kann gelten, dass ein niedriger CRP-Wert zusammen mit einer nur geringen Symptomatik eine Pneumonie unwahrscheinlich macht. Die unnötige Gabe von Antibiotika bei mutmaßlich viralen Infekten kann so reduziert werden [10]. Aufgrund des verzögerten Anstiegs im Initialstadium der Pneumonie (6-12 Stunden) und niedrigeren Werten bei immundefizienten und älteren Patienten ist jedoch Vorsicht geboten. Patienten mit niedrigem CRP-Wert, aber ausgeprägter pulmonaler Symptomatik sollten zum Ausschluss einer Pneumonie eine Röntgen-Thoraxaufnahme erhalten ( Tab. 2).

Die Serummessung des CRP kann als Parameter zum Ausschluss, zur Diagnose und zum Verlauf einer Pneumonie genutzt werden. Niedrige CRP-Werte sprechen gegen eine CAP, die CRP-Bestimmung ist daher insbesondere hilfreich in der Abgrenzung der CAP zur akuten Bronchitis. Der Nachteil der Untersuchung liegt in der niedrigen Spezifität und der verzögerten Kinetik mit einem Anstieg im Mittel erst nach 6 Stunden.

Eine CRP-Erhöhung trägt zur Diagnose einer CAP bei, da erhöhte CRP-Werte sensitiver und spezifischer sind als Temperaturerhöhung oder Leukozytose [11]. In verschiedenen Studien war ein $\mathrm{Cu}$ toff von $100 \mathrm{mg} / \mathrm{l}$ geeignet, um mit relativ hoher
Spezifität die CAP von der infektexazerbierten COPD oder anderen pulmonalen Erkrankungen zu unterscheiden [12]. Leicht erhöhte Werte differenzieren jedoch nicht zwischen viralen und bakteriellen Infekten. Nennenswert ist zudem, dass die differenzialdiagnostisch infrage kommenden Erkrankungen ebenfalls mit einer CRPErhöhung einhergehen können. Ein höherer CRPWert spricht nicht unbedingt für eine schwerere Erkrankung. Garcia Vazquez u. Mitarb. fand bei 1222 Patienten mit CAP keinen Zusammenhang zwischen dem Schweregrad der CAP und dem CRP-Wert [13]. Seppa u. Mitarb. konnten hingegen zeigen, dass bei älteren Patienten (>65 J.) mit CAP ein CRP > $100 \mathrm{mg} / \mathrm{L}$ mit einer erhöhten Mortalität verbunden ist [14]. Bemerkenswert ist, dass Patienten mit Pneumokokken-Pneumonie und Legionellen-Pneumonie im Durchschnitt höhere CRP-Werte aufweisen [13,14].

Das CRP kann für die Dokumentation des Therapieerfolgs verwendet werden.

Falls das CRP nicht innerhalb von 4 Tagen auf $50 \%$ fällt, spricht dies für eine Therapieversagen oder für ein sekundäre infektiöse Komplikation (z.B. Empyem, Endokarditis, Antibiotika assoziierte Diarrhoe, Harnwegsinfekt) [11].

Prokalzitonin. Virale und nichtinfektiöse Erkrankungen können ebenfalls zu einer CRP-Erhöhung führen. Eine mögliche Verbesserung in dieser Hinsicht stellt das Prokalzitonin (PCT) dar. Das PCT ist die Vorform des Kalzitonin, welches als Reaktion auf eine Hyperkalzämie in der Schilddrüse gebildet wird. Das PCT ist normalerweise im Blut nicht messbar. Bakterielle Antigene führen während bakterieller Infektionen durch Induktion von Zytokinen zu einer extrathyreoidalen Produktion von PCT in der Leber und den mononukleären Zellen. Zirkulierendes PCT ist während schwerer bakterieller Infektionen erhöht, jedoch niedrig bei viralen Infektionen und nicht infektiösen Inflammationen [15]. Falsch positive PCT-Werte sind z.B. während des Gichtanfalls oder postoperativ beschrieben. Das Ziel einer gut durchgeführten Studie [16] war die Reduktion der Antibiotikagabe bei Patienten mit Infektion des unteren Respirationstraktes. Es wurde gezeigt, dass eine PCT-gesteuerte Therapieentscheidung die Wahrscheinlichkeit einer Antibiotikatherapie drastisch reduziert, ohne die Prognose zu verschlechtern [16]. Patienten mit einer niedrigen PCT-Konzentration benötigen daher möglicherweise keine Antibiotikatherapie. Größere Studien werden derzeit durchgeführt. Bestätigen sich die Befunde, könnte dieser Marker
Tab. 2 Die Rolle des CRP in der Pneumoniediagnostik 
Abb. 2 Ätiologie der ambulant erworbenen Pneumonie (CAP): mikrobiologische Ergebnisse aus CAPNETZ (www.capnetz.de)

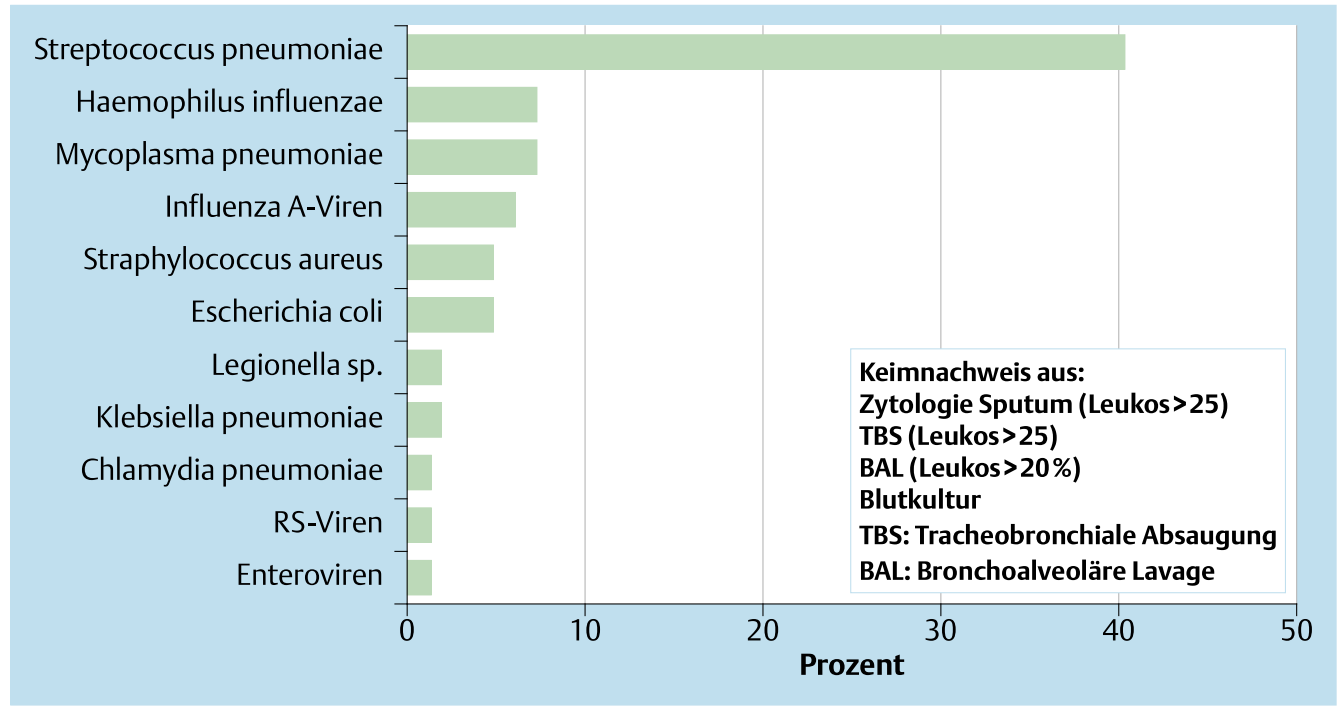

helfen, unnötige Antibiotikagaben zu vermeiden, insbesondere bei COPD-Exazerbationen und Patienten mit akuter Bronchitis (bei denen normalerweise keine Antibiotika indiziert sind).

Differenzialdiagnostisch schwierig bleiben Patienten mit einer pulmonalen Symptomatik, radiologisch nachgewiesenem Infiltrat, aber niedrigem PCT oder CRP. Diese haben möglicherweise eine virale CAP, die nach Expertenmeinung prophylaktisch mit Antibiotika behandelt werden sollte, eine bakterielle CAP bei einem noch nicht angestiegenen Entzündungswert oder ein nichtinfektiöses Infiltrat.

Die neueren Daten zur PCT-Messung sind vielversprechend. Das PCT ist spezifischer als das CRP und steigt bereits nach 4 Stunden an. Die Kosteneffektivität ist noch nicht untersucht. Die Ergebnisse aktuell laufender Studien sollten abgewartet werden, bevor PCT in der Praxis eingesetzt wird.

\section{Erregerdiagnostik}

Der Sinn der mikrobiologischen Diagnostik kann entweder darin liegen, einen Erreger zu entdecken, der zu einer Erweiterung der Antibiotikatherapie führt (z.B. Legionellen, Staphylococcus aureus oder gramnegative Bakterien), oder um bei Nachweis eines bestimmten Keimes (z.B. Pneumokokken) eine gezieltere Antibiotikatherapie durchzuführen ( Abb. 2). Der Nachweis einer Bakteriämie hat eine prognostische Bedeutung. Indikation zur mikrobiologischen Diagnostik mittels Sputum oder Bronchoskopie siehe

Tab. 3.
Die routinemäßige mikrobiologische Diagnostik mittels Sputum oder Bronchoskopie beeinflusst die Therapie nicht und wird nicht empfohlen [1]. Bei Patienten mit schwerer Pneumonie ist eine initiale mikrobiologische Diagnostik sinnvoll. Der Nutzen einer Bronchoskopie muss gegen die möglichen Risiken (Zunahme der respiratorischen Insuffizienz, Notwendigkeit der maschinellen Beatmung) abgewogen werden. Bei Patienten mit Immundefizit ist in der Regel eine bronchoskopische Erregergewinnung sinnvoll [17]. Bei rezidivierender oder therapierefraktärer Pneumonie ist neben der mikrobiologischen Diagnostik häufig eine Differenzialdiagnostik nötig ( Tab. 4), so dass die Bronchoskopie meist sinnvoller als die Sputumuntersuchung ist.

\section{Sputumdiagnostik (Sputumkultur und Gramfärbung)}

Die Qualität der Sputumdiagnostik hängt wesentlich von der Infrastruktur der Institution und der Expertise des Untersuchers ab [18]. Ein Problem sind die häufig falsch positiven Befunde bei Kontamination durch Rachenflora. Die Häufigkeit eindeutiger Sputumbefunde ist daher in den letzten Jahrzehnten drastisch gesunken [19]. Untersucht werden sollte nur makroskopisch eitriges Sputum (25 Neutrophile und weniger als 10 Plattenepithelzellen pro 100fach vergr. Gesichtsfeld) innerhalb einer Verarbeitungszeit von 2-4 Stunden. Das mikrobiologische Labor sollte Material ohne entsprechende Qualität nicht untersuchen. Der Anteil von qualitativ guten Sputen mit prädominierenden Morphotyp liegt jedoch nur bei $5-15 \%[20,21]$. Bei bis zu $80 \%$ der unter-
Tab. 3 Diagnostik mittels Sputum oder Bronchoskopie
Sputum generell nur bei geeigneter Infrastruktur

bei unkomplizierter CAP Sputum oder Bronchoskopie nicht sinnvoll

bei schwerer Pneumonie qualitativ „gutes“ Sputum oder primäre Bronchoskopie sinnvoll

bei rezidivierender, therapierefraktärer, poststenotischer CAP Bronchoskopie sinnvoll

bei Immunsuppression oder Tuberkuloseverdacht (neg. Sputum) Bronchoskopie sinnvoll

* cave respiratorische Verschlechterung durch Bronchoskopie 
suchten Sputen kann der Erreger in der Gramfärbung nachgewiesen werden. Eine eindeutige Gramfärbung kann Einfluss haben auf die Therapie [22].

Die Sputumdiagnostik ist bei guter Infrastruktur und in geübten Händen eine sensitive Methode. Im ambulanten Bereich und auch in vielen Krankenhäusern ist in der Regel die Infrastruktur nicht gegeben, so dass hier der Einsatz nicht gerechtfertigt ist.

\section{Blutkultur}

Der Nachweis einer Bakteriämie mittels Blutkultur ist sehr spezifisch und gelingt am häufigsten bei Streptococcus pneumoniae, Escherichia coli, Hämophilus influenzae, Staphylococcus aureus und Klebsiella pneumoniae. Die Bakteriämie ist ein Marker für die Schwere der Erkrankung. GröBere Studien zeigen, dass etwa 10\% der hospitalisierten CAP-Patienten bakteriämisch sind. Bei Patienten mit Pneumokokkenpneumonie liegt die Rate bei etwa $25 \%$, bei Patienten mit antibiotischer Vortherapie niedriger. Die Chance einer positiven Blutkultur ist abhängig vom Schweregrad mit einer Nachweisrate von $25 \%$ bei schwerer Pneumonie [23]. Eine positive Blutkultur führt jedoch nur selten zu einer Änderung des therapeutischen Regimes [24].

Die Blutkultur ist nur bei hospitalisierten, schwerkranken Patienten mit ambulant erworbener Pneumonie sinnvoll. Bei Patienten mit unkomplizierter Pneumonie ohne Komorbidität kann auf eine Blutkultur verzichtet werden.

\section{Diagnostik einzelner Erreger}

Streptococcus pneumonia. Pneumokokken-Antigene können in Sputum, Pleuraflüssigkeit, Serum, Urin und Liquor nachgewiesen werden. Der neueste, vielversprechende Pneumokokken-Antigentest ist ein Immunchromatographie-Membran-Test (ICT) und detektiert Zellwand-Polysaccharide (Binax NOW). Er kann „bedside“ mit Urin innerhalb von ca. 15 min durchgeführt werden. Eine vorherige Konzentration des Urins wird nicht empfohlen. Verglichen mit der Blutkultur und der Sputumkultur hat der Test eine etwa 60-80\%-Sensitivität mit einer Spezifität von etwa 90\% [25]. Die Ausbeute ist höher bei Bakteriämie und Patienten mit schwerer Pneumonie [25]. Guiterez u. Mitarb. untersuchten bei 452 CAP Patienten den Test in konzentriertem Urin [26]. Bei 19 (70\%) von 27 kulturell gesicherten Pneumokokkenpneumonien war der Test positiv. Zusätzlich hatten 69 (29\%) der Patienten ohne Erregernachweis einen positiven Test, so dass möglicherweise bei einem großen Anteil bisher nicht definierter Pneumonien Pneumokokken als Erreger nachgewiesen werden könnten. Allerdings waren 16 (10\%) von 156 Proben von Patienten mit kulturellem Nachweis eines

\section{Ursachen \\ Keime, die nicht auf die kalkulierte Therapie ansprechen: z. B. Legionellen, Chlamydien, \\ Mykoplasmen, Pseudomonas, Viren, TB, \\ Aspergillen \\ Superinfektion mit resistenten Keimen: \\ z. B. MRSA \\ Neoplasie der Lunge \\ Lungenarterienembolie \\ Herzinsuffizienz \\ Lungenfibrose \\ bisher nicht erkannte HIV-Infektion \\ Pneumozystis-Infektion, TB, selten CMV}

anderen Erregers ebenfalls positiv, so dass die Spezifität in Abhängigkeit vom Patientenkollektiv begrenzt ist. Bei Kleinkindern ist die Rate an positiven Tests bei nasopharyngealer Kolonisation sehr hoch. Bei Erwachsenen kann insbesondere bei COPD ein falsch positiver Test vorkommen [27]. Wichtig ist, dass der Test in 50\% der Patienten auch nach 6 Wochen noch positiv ist [27]. Ein wesentlicher Vorteil liegt darin, dass der Antigentest auch bei antibiotisch vorbehandelten Patienten noch positiv sein kann. Ein Nachteil ist die fehlende Möglichkeit der Resistenztestung.

Der Nachweis des Antigens im Urin erlaubt bisher keine sichere Fokussierung der Therapie auf Pneumokokken, da der Test falsch positiv sein kann und eine Mischinfektion nicht ausgeschlossen ist. Ein negativer Test hingegen schließt eine Pneumokokken-Pneumonie nicht sicher aus. Der Pneumokokken-Antigentest wird daher nicht allgemein empfohlen.

Legionella pneumophila. Die Legionellenpneumonien sind in Deutschland seltene $1-8 \%$ der CAP) umweltbedingte Infektionen bei typischen Risikofaktoren (Komorbidität, Steroidtherapie, Immunsuppression, Fernreisen, Exposition/Wasser, Versagen einer Betalaktamtherapie, Epidemien). Zum Nachweis kann der LegionellenUrin-Antigentest dienen. Kommerzielle Kits auf der Basis von Enzymimmunoassays (EIA) oder Immunochromatographieassays (ICT) sind verfügbar ( Abb.3). Die Spezifität liegt bei $99-100 \%$ mit einer Sensitivität von $76-94 \%$ [28]. Die Assays detektieren nur Legionella pneumophila Serotyp 1, welcher ca. 80\% der Legionellosen verursacht. Die Urinkonzentrierung erhöht die Sensitivität. Der Test hat bei schwerer Pneumonie eine höhere Sensitivität und hatte in verschiedenen Studien einen Einfluss auf die primäre Antibiotikatherapie und die Prognose [29].

Der Legionellen-Urin-Antigentest ist sinnvoll bei allen Patienten mit:

schwerer Pneumonie

Risikofaktoren

Therapieversagen unter Betalaktamtherapie. Ein weiteres Nachweisverfahren für Legionellen ist die direkte Immunfluoreszenz (DIF) in bronchialen Sekreten. Sie wird wegen geringer Sensi-
Tab. 4 Typische Differenzialdiagnosen der therapierefraktären CAP 
Abb. 3 Immunochromatographischer Membranassay (Binax NOW $^{\circledR}$ ) zum Nachweis von Legionella pneumophila Antigen der Serogruppe $1 \mathrm{im}$ Urin bestehend aus der Testmembran, einem Abstrichtupfer und Reagens A (A). Der Tupfer wird nach Eintauchen in den Urin in das Testbesteck gesteckt und Reagens A wird hinzugegeben. Durch Schließen des Testbestecks kommt die Probe in Kontakt mit auf der Testmembran immobilisierten Anti-L.-Pneumophila-Serogruppe 1 Antikörpern. Bei Antigen-positiven Proben werden konjugierte Antikörper gebunden, so dass sich 15 min nach Testbeginn neben der Kontrollbande eine weitere Bande im Sichtfenster zeigt (B).

Tab. 5 Relevanz der gefundenen Erreger

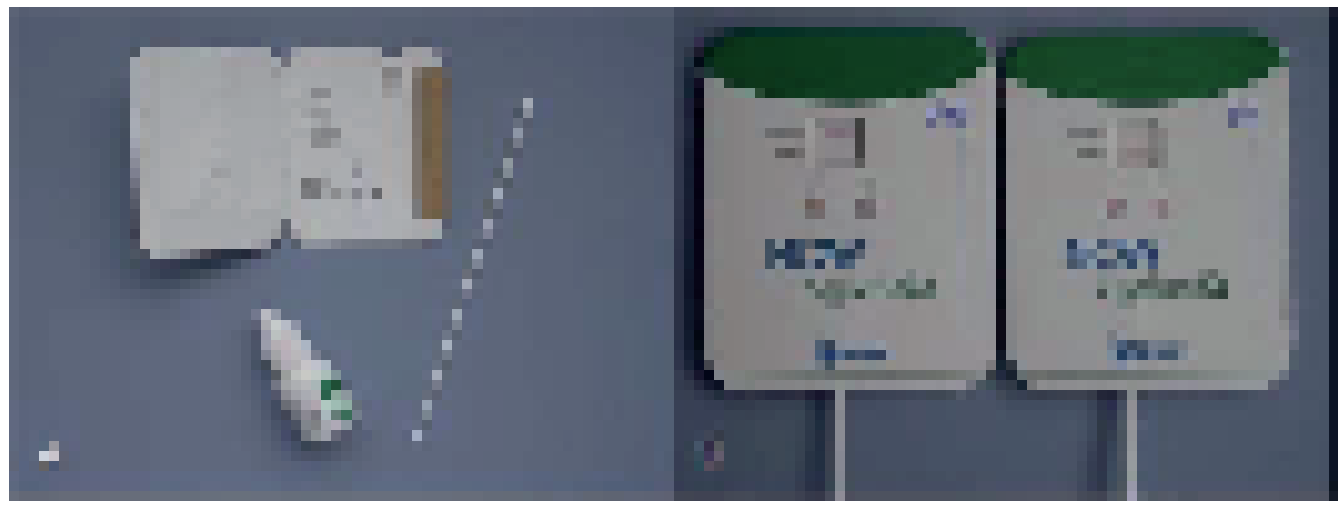

tivität nicht empfohlen. Die Kultur aus Sputum oder bronchoalveolärer Lavage (BAL) ist zwar hochspezifisch, die Sensitivität mit 40\% gering und dauert mit bis zu 10 Tagen zu lang [30]. Die Legionellenserologie ist erst bei 4 fachem Titeranstieg (2-6 Wochen) beweisend. Der IgM Nachweis ist nicht validiert, insbesondere da die Antikörper über Jahre persistieren können. Die PCRMethoden sind vielversprechend, aber nicht validiert.

Indikationen für den Legionellen-Urin-Antigentest sind: Schwere Pneumonien, Risikofaktoren und Pneumonieversagen. Die Serologie und die Legionellen-Kultur sind nur für epidemiologische Zusammenhänge sinnvoll. Der Immunfluoreszenz-Test und die PCR werden nicht empfohlen.

Tests bei Verdacht auf Chlamydien, Mykoplasmen oder Viren

Bei Verdacht auf Chlamydia psittaci-Infektionen kann eine Serologie in spezialisierten Laboren durchgeführt werden. Bei Verdacht auf Chlamydophila pneumoniae ist aufgrund der möglichen Fehlerquellen (Reinfektion, Persistenz des Erregers etc.) die Serologie und der Mikroimmunfluoreszenz-MIF-Test nicht hilfreich [1]. Die PCR aus respiratorischen Sekreten wird nicht routinemäßig angeboten, kann aber am ehesten eine akute Infektion wahrscheinlich machen.

Bei Verdacht auf Mycoplasma pneumoniae sind die kulturellen Verfahren wegen niedriger Sensitivität und einer Dauer von 10-14 Tagen für die Klinik nicht geeignet. Enzymimmunoassays zum Nachweis von IgM-Antikörpern in der Frühphase der Infektion sind spezifisch (Spezifität $>95 \%$ ), die Sensitivität ist mit ca. $50 \%$ jedoch niedrig, kann aber durch eine Verlaufsuntersuchung er- höht werden [31]. Durch zusätzliche IgA-Bestimmung sollte das Ergebnis gegenüber einem persistierenden IgM abgegrenzt werden. Der Direktnachweis mittels Real-Time-PCR aus respiratorischem Material ist derzeit die schnellste und verlässlichste Methode zum Nachweis der akuten $M$. pneumoniae Infektion [32].

Für Influenzaviren stehen Antigen-Schnelltests für respiratorische Materialien mit einer Sensitivität von $70-90 \%$ zur Verfügung. Die Antigentests für Respiratory Syncytial Virus (RSV) haben lediglich eine Sensitivität von 15\%. Die Direkte Immunfluoreszenz und PCR ist möglich für Influenza-, Parainfluenza-, Adeno- und RS-Viren, valide Daten existieren jedoch nicht. Bei V.a. Coxiella burnetti Infektion ist eine Serologie in Speziallaboren möglich.

\section{Routinemäßige Untersuchungen auf Chlamydien, Myko- plamen und Viren sind nicht sinnvoll. Wenn überhaupt wäre für Chlamydophila und für Mykoplasmen die PCR am ehesten geeignet, eine Infektion nachzuweisen. Bei Aus- bruchssituationen, aktuell auch bei H5N1 ist der Influen- zanachweis mittels Antigenschnelltest sinnvoll.}

\section{Interpretation von Erregernachweisen}

Für die Interpretation der Befunde ist entscheidend, ob der Patient eine klinisch und radiologisch definierte CAP hat und welcher Erreger nachgewiesen wurde. Obligat pathogene Keime sind in $\bullet$ Tab. 5 aufgeführt. Fakultativ pathogene Keime wie Pneumokokken oder Hämophilus sind bei Patienten mit nachgewiesener Pneumonie als relevant anzusehen. Der Nachweis dieser Erreger in Bronchialsekreten ohne Infiltrat und Entzündungszeichen spricht hingegen für eine Besiedelung (๑ Tab.5).

\begin{tabular}{|lll|}
\hline $\begin{array}{l}\text { Obligat pathogen } \\
\text { Legionella spp. }\end{array}$ & Fakultativ pathogen & Selten pathogen \\
\hline Mycoplasma pneumoniae & Streptococcus. pneumoniae & S. viridans \\
\hline Influenzaviren & Haemophilus influenza & Enterokokken \\
\hline & Chlamydophila pneumoniae & Neisserien \\
\hline & Pseudomonas aeruginosa & \\
\hline Mycobacterium tuberculosis & gramneg. Enterobakterien & \\
\hline Pneumocystis jiroveci & atypische Mykobakterien & \\
\hline
\end{tabular}




\begin{tabular}{|c|c|}
\hline Untersuchung & Bewertung \\
\hline \multicolumn{2}{|l|}{ Apparative Diagnostik } \\
\hline Röntgen-Thoraxaufnahme & sinnvoll zur Diagnosesicherung + Ausschluss DD \\
\hline Computertomographie der Thoraxorgane & $\begin{array}{l}\text { bei Komplikationen + Ausschluss DD (Tumor, Embolie, entzünd- } \\
\text { lichen Lungenerkrankungen) }\end{array}$ \\
\hline Bronchoskopie & $\begin{array}{l}\text { bei Therapieresistenz oder Immundefizit zur Keimgewinnung + } \\
\text { Ausschluss DD incl. TB }\end{array}$ \\
\hline \multicolumn{2}{|l|}{ Entzündungsdiagnostik } \\
\hline CRP & $\begin{array}{l}\text { sinnvoll zur Diagnosesicherung und Verlaufsuntersuchung } \\
\text { (Nachteil: unspezifisch) }\end{array}$ \\
\hline Prokalzitonin (PCT) & in Zukunft möglicherweise besser als CRP, da spezifischer \\
\hline \multicolumn{2}{|l|}{ Mikrobiologie } \\
\hline Sputum: Gram Fbg. + Kultur & $\begin{array}{l}\text { nur bei guter Infrastruktur } \\
\text { ansonsten zum TB-Ausschluss }\end{array}$ \\
\hline Blutkultur & nur bei schwerkranken, hospitalisierten Patienten \\
\hline Serologie & bei V.a. Epidemien und Einzelfälle \\
\hline Pneumokokken-Urinantigen & bisher noch nicht empfohlen \\
\hline Legionellen-Urinantigen & sinnvoll bei schwerer Pneumonie und Risikopatienten \\
\hline \multicolumn{2}{|l|}{ Serum } \\
\hline HIV-Antikörpertest & sinnvoll bei jüngeren Patienten \\
\hline
\end{tabular}

Der Nachweis von Pseudomonaden bei Patienten mit CAP sollte zu einer entsprechenden Antibiotikatherapie führen.

Sowohl die Candida-Infektion als auch Infektionen mit z.B. E. coli entstehen typischerweise multifokal bei hämatogener Streuung. Der Nachweis dieser Keime in bronchialen Sekreten sollte daher in Zusammenhang mit Klinik und dem Röntgenbefund gesehen werden. Candida spp. werden bei tracheobronchialer Besiedlung häufig gefunden und sind meist ohne therapeutische Konsequenz. E. coli, andere gramneg. Enterobakterien und Enterokokken sind typische Kontaminationskeime und häufig nicht relevant.

\section{Fazit für die Praxis}

Die bei ambulant erworbener Pneumonie notwendige Diagnostik ist in Tab. 6 aufgeführt. Um unnötige Antibiotikatherapien zu vermeiden, sollte der behandelnde Arzt eine definitive Diagnose der Pneumonie stellen. Wir empfehlen daher vor der Antibiotikatherapie eine RöntgenThoraxaufnahme und eine Messung des CRP durchzuführen. Eine Erregerdiagnostik ist im ambulanten Bereich nur in Ausnahmefällen nötig. Bei hospitalisierten Patienten sollte nur bei entsprechender Infrastruktur eine Sputumuntersuchung durchgeführt werden. Die Bronchoskopie ist sinnvoll bei Therapieresistenz zur Keimgewinnung und in der Differenzialdiagnostik. Bei Patienten mit Risiko für Legionellen-Infektion sollte der Urin-Antigentest verwendet werden. Eine Blutkultur hingegen ist nur bei schwerkranken Patienten sinnvoll ( Tab.6).

\section{Literatur}

1 Höffken G, Lorenz J, Kern W et al. S3-guideline on ambulant acquired pneumonia and deep airway infections. Pneumologie 2005; 59: 612-664

2 Metlay JP, Schulz R, Li YH et al. Clinical prediction rule for pulmonary infiltrates. Ann Intern Med 1990; 113: 664 670

3 Young M, Marrie TJ. Interobserver variability in the interpretation of chest roentgenograms of patients with possible pneumonia. Arch Intern Med 1994; 154: 2729-2732

4 Kauppinen MT, Lahde S, Syrjala H. Roentgenographic findings of pneumonia caused by Chlamydia pneumoniae. A comparison with streptococcus pneumonia. Arch Intern Med 1996; 156: 1851- 1856

5 Garcia-Ordonez MA, Garcia-limenez IM et al. Clinical aspects and prognostic factors in elderly patients hospitalised for community-acquired pneumonia. Eur J Clin Microbiol Infect Dis 2001; 20: 14-19

6 Woodhead MA, Macfarlane JT, McCracken JS et al. Prospective study of the aetiology and outcome of pneumonia in the community. Lancet 1987; 1: 671-674

7 Syrjala H, Broas M, Suramo I et al. High-resolution computed tomography for the diagnosis of communityacquired pneumonia. Clin Infect Dis 1998; 27: 358-363

8 Fine MJ, Smith MA, Carson CA et al. Prognosis and outcomes of patients with community-acquired pneumonia. A meta-analysis. JAMA 1996; 275: 134-141

9 Almirall J, Bolibar I, Toran P et al. Contribution of C-reactive protein to the diagnosis and assessment of severity of community-acquired pneumonia. Chest 2004; 125 : $1335-1342$

10 Hopstaken RM, Muris JW, Knottnerus JA et al. Contributions of symptoms, signs, erythrocyte sedimentation rate, and C-reactive protein to a diagnosis of pneumonia in acute lower respiratory tract infection. $\mathrm{Br} J$ Gen Pract 2003; 53: $358-364$

11 Hansson LO, Hedlund JU, Ortqvist AB. Sequential changes of inflammatory and nutritional markers in patients with community-acquired pneumonia. Scand J Clin Lab Invest 1997; 57: $111-118$

12 Castro-Guardiola A, Armengou-Arxe A, Viejo-Rodriguez A et al. Differential diagnosis between community-acquired pneumonia and non-pneumonia diseases of the chest in the emergency ward. Eur J Intern Med 2000; 11: $334-339$

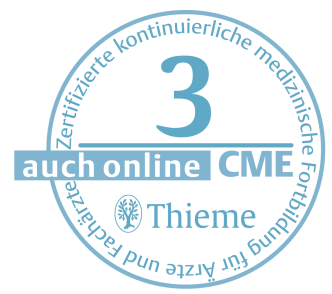


13 Garcia Vazquez E, Martinez JA, Mensa J et al. C-reactive protein levels in community-acquired pneumonia. Eur Respir J 2003; 21: 702 - 705

14 Seppa Y, Bloigu A, Honkanen PO et al. Severity assessment of lower respiratory tract infection in elderly patients in primary care. Arch Intern Med 2001; 161: $2709-2713$

15 Muller CA, Uhl W, Printzen $G$ et al. Role of procalcitonin and granulocyte colony stimulating factor in the early prediction of infected necrosis in severe acute pancreatitis. Gut 2000; 46: $233-238$

16 Christ-Crain M, Jaccard-Stolz D, Bingisser $R$ et al. Effect of procalcitonin-guided treatment on antibiotic use and outcome in lower respiratory tract infections: clusterrandomised, single-blinded intervention trial. Lancet 2004; 363: 600-607

17 Dalhoff K, Ewig S, Höffken G et al. Recommendations for the diagnosis, therapy and prevention of pneumonia in the immunocompromised host. Pneumologie 2002; 56: $807-831$

18 Reed WW, Byrd GS, Gates Jr RH et al. Sputum gram's stain in community-acquired pneumococcal pneumonia. A meta-analysis. West J Med 1996; 165: 197 - 204

19 Bartlett JG. Decline in microbial studies for patients with pulmonary infections. Clin Infect Dis 2004; 39: $170-$ 172

20 Theerthakarai R, El-Halees W, Ismail M et al. Nonvalue of the initial microbiological studies in the management of nonsevere community-acquired pneumonia. Chest 2001; 119: 181 - 184

21 Garcia-Vazquez E, Marcos MA, Mensa J et al. Assessment of the usefulness of sputum culture for diagnosis of community-acquired pneumonia using the PORT predictive scoring system. Arch Intern Med 2004; 164: $1807-1811$

22 Roson B, Carratala J, Verdaguer $R$ et al. Prospective study of the usefulness of sputum Gram stain in the initial approach to community-acquired pneumonia requiring hospitalization. Clin Infect Dis 2000; 31: 869-874

23 Waterer GW, Wunderink RG. The influence of the severity of community-acquired pneumonia on the usefulness of blood cultures. Respir Med 2001; 95: 78-82
24 Corbo J, Friedman B, Bijur P et al. Limited usefulness of initial blood cultures in community acquired pneumonia. Emerg Med J 2004; 21: 446-448

25 Roson B, Fernandez-Sabe N, Carratala J et al. Contribution of a urinary antigen assay (Binax NOW) to the early diagnosis of pneumococcal pneumonia. Clin Infect Dis 2004; 38: $222-226$

26 Gutierrez F, Masia M, Rodriguez JC et al. Evaluation of the immunochromatographic Binax NOW assay for detection of Streptococcus pneumoniae urinary antigen in a prospective study of community-acquired pneumonia in Spain. Clin Infect Dis 2003; 36: 286- 292

27 Murdoch DR, Laing RT, Cook JM. The NOW S. pneumoniae urinary antigen test positivity rate 6 weeks after pneumonia onset and among patients with COPD. Clin Infect Dis 2003; 37: $153-154$

28 Helbig JH, Uldum SA, Bernander S et al. Clinical utility of urinary antigen detection for diagnosis of communityacquired, travel-associated, and nosocomial legionnaires' disease. J Clin Microbiol 2003; 41: 838 - 840

29 Lim WS, Macfarlane JT, Boswell TC et al. Study of community acquired pneumonia aetiology (SCAPA) in adults admitted to hospital: implications for management guidelines. Thorax 2001; 56: 296-301

30 Lindsay DS, Abraham WH, Findlay W et al. Laboratory diagnosis of legionnaires' disease due to Legionella pneumophila serogroup 1: comparison of phenotypic and genotypic methods. J Med Microbiol 2004; 53: $183-$ 187

31 Beersma MF, Dirven K, Dam AP van et al. Evaluation of 12 commercial tests and the complement fixation test for Mycoplasma pneumoniae-specific immunoglobulin $G$ (IgG) and IgM antibodies, with PCR used as the "gold standard”. J Clin Microbiol 2005; 43: 2277- 2285

32 Templeton KE, Scheltinga SA, Graffelman AW et al. Comparison and evaluation of real-time PCR, real-time nucleic acid sequence-based amplification, conventional $P C R$, and serology for diagnosis of Mycoplasma pneumoniae. J Clin Microbiol 2003; 41: 4366-4371

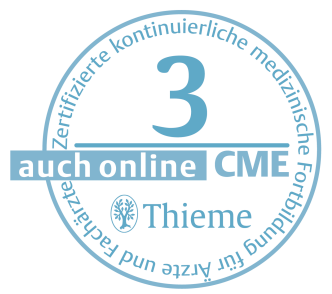




\section{CME-Fragen Aktuelle Diagnostik der ambulant erworbenen Pneumonie}

1 Die Diagnose CAP bei einem 45-jährigen Patienten mit Husten, vermehrt Sputum, verstärkter Atemarbeit und Fieber

A sollte ohne Blutuntersuchung und Röntgen-Thoraxaufnahme gestellt werden.

B sollte mittels CT-Thorax bestätigt werden.

C ist, damit die Diagnose abgerechnet werden kann, mikrobiologisch zu bestätigen.

D sollte durch eine Röntgen-Thoraxaufnahme bestätigt werden um unnötige Antibiotikatherapien bei akuter Bronchitis zu vermeiden.

E ist ausgeschlossen, da der Patient keine Hypotonie aufweist.

2 Die Röntgen-Thoraxaufnahme bei Verdacht auf CAP

A ist spezifisch bei Infektion mit Legionellen.

B spricht bei Nachweis eines Infiltrates für eine Pneumonie, hat aber keine Aussagekraft für die Prognose.

C sollte bei unkomplizierten Pneumonien erst nach 10 Tagen wiederholt werden.

D sollte zur Diagnose und/oder Ausschluss einer Pneumonie eingesetzt werden.

E sollte bei älteren Patienten seltener durchgeführt werden.

3 Das CRP wird zur Pneumoniediagnostik genutzt, welche Aussage trifft am ehesten zu?

A Ein CRP von $10 \mathrm{mg} / \mathrm{l} \mathrm{schließt}$ eine bakterielle Pneumonie definitiv aus.

B Ein CRP von $100 \mathrm{mg} / \mathrm{l}$ beweist eine bakterielle Pneumonie.

C Legionellen Infektionen haben häufig besonders hohe CRP-Werte.

D Die Kombination CRP von $50 \mathrm{mg} / \mathrm{l}$ und Infiltrat ist beweisend für die CAP.

E Die Kombination CRP von $150 \mathrm{mg} / \mathrm{l}$ und Infiltrat ist beweisend für die CAP.

4 Ein 64-jähriger Patient (Raucher) hat 3 Wochen Husten und Auswurf mit Temperaturen bis 38 C. Ein Therapie mit Amoxicillin + Clavulansäure p. o. war nicht effektiv. Radiologisch zeigt sich ein Oberlappeninfiltrat links. Welche Aussage ist falsch?

A Eine Röntgen-Thoraxaufnahme ist sinnvoll.

B Auf die Bestimmung der Entzündungswerte sollte nicht verzichtet werden

C Atypische Erreger sind wahrscheinlich (therapeutische Lücke), daher sollte eine mikrobiologische Diagnostik mit Mykoplasmen und Chlamydien Serologie erfolgen.

D Eine Bronchoskopie und ein CT-Thorax ist sinnvoll, da differenzialdiagnostisch eine Neoplasie infrage kommt.

E Eine Sputumuntersuchung hilft lediglich in Einzelfällen bei guter Infrastruktur.
5 Welche Aussage trifft zu?

A Legionellen können kulturell nicht angezüchtet werden, daher sollte der Nachweis mittels Urin-Antigentest erfolgen.

B Der Urin-Antigentest wird nur in Speziallaboren durchgeführt.

c Therapieversagen mit Tetracyclinpräparaten sollte an Legionelleninfektionen denken lassen.

D Der Legionellen-Urin-Antigentest weist lediglich Serotyp 1 nach.

E Ein positiver Urin-Antigentest sollte mittels Serologie bestätigt werden.

Ein 62-jähriger Patient mit Husten und Auswurf berichtet über Temperatur von $39 \mathrm{C}$, hat jedoch keine Dyspnoe: Auskultation o. B.; CRP 145 mg/l, Infiltrat links basal in der Röntgen-Thoraxaufnahme. Welche Aussage ist richtig?

A Ene Antibiotikatherapie ist nicht indiziert, da der Patient ohne Dyspnoe keine Pneumonie haben kann.

B Eine Antibiotikatherapie ist nicht indiziert bei einem CRP unter $150 \mathrm{mg} / \mathrm{l}$, da es sich wahrscheinlich um eine virale Pneumonie handelt.

C Eine Antibiotikatherapie ist sinnvoll aufgrund des Infiltrates in der Röntgen-Thoraxaufnahme, der Klinik des Patienten und der stark erhöhten CRP-Werte, andere Differenzialdiagnosen sind aber nicht ausgeschlossen.

D Da das CRP spezifisch für Infektionen ist, kann eine andere Genese des Infiltrates (z. B. Embolie) ausgeschlossen werden.

E Ein CRP von $145 \mathrm{mg} / \mathrm{l}$ bei Nachweis eines Lobärinfiltrats schließt eine Infektion mit intrazellulären Erregern (z.B. Mykoplasmen-Pneumonie) aus.

Ein 30-jähriger Patient mit Temp. 38 C, Auskultation o. B. $\mathrm{pO}_{2} 55 \mathrm{~mm} \mathrm{Hg}$, bds. perihiläre Infiltrate, Klarithromycin war ohne Effekt. Welche Differenzialdiagnose ist am wenigsten wahrscheinlich?

A Mykoplasmen-Pneumonie

B Makrolid resistente Pneumokokken

C Bisher unerkannte HIV-Infektion und Pneumozystose der Lunge

D Beidseitige Lungenembolie

E Herzinsuffizienz 
Welche Aussage trifft zu?

A Die Bestimmung des CRP ist ein spezifische Marker für bakterielle Infektionen.

B Die Bestimmung des PCT ist etabliert und sollte anstatt der CRP-Messung eingesetzt werden.

C Die Bestimmung des PCT hilft möglicherweise unnötige Antibiotikagaben einzusparen.

D PCT-Werte in der Infektiologie zu bestimmen ist nicht sinnvoll, da es sich um einen endokrinologischen Parameter handelt.

E PCT ist erhöht bei viralen Infektionen und hilft daher Influenzapneumonien zu erfassen.

9 Welche Aussage trifft nicht zu?

A Die Entnahme von Blutkulturen ist bei unkomplizierter CAP in der Regel nicht hilfreich.

B Die Bakteriämie ist ein Marker für die Schwere der Erkrankung.

C Bei schweren Pneumonien ist die Rate positiver Blutkulturen 25\%.

D Eine positive Blutkultur führt nur selten zu einer Änderung des therapeutischen Regimes.

E Mykoplasmen und Chlamydien lassen sich besonders gut in der Blutkultur nachweisen.
Der Nachweis von reichlich E. coli im Sputum (10 Neutrophile und 25 Plattenepithelzellen pro 100fach vergr. Gesichtsfeld) nach Therapie einer CAP mit Amoxicillin spricht für

A Superinfektion.

B Sekundär-Resistenz.

C Superspreader.

D Kontamination mit Rachenflora.

E Mischinfektion.

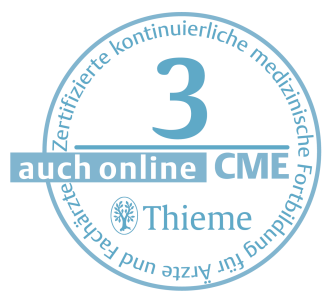




\section{A Angaben zur Person}

\section{B Lernerfolgskontrolle} ankreuzen

Bitte nur eine Antwort pro Frage

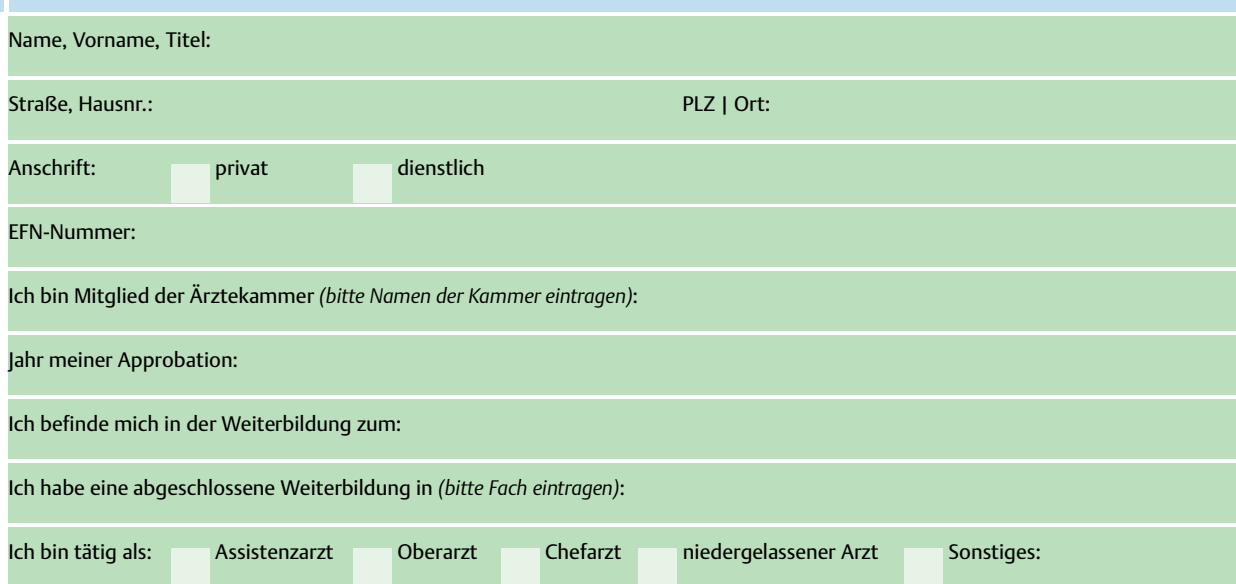
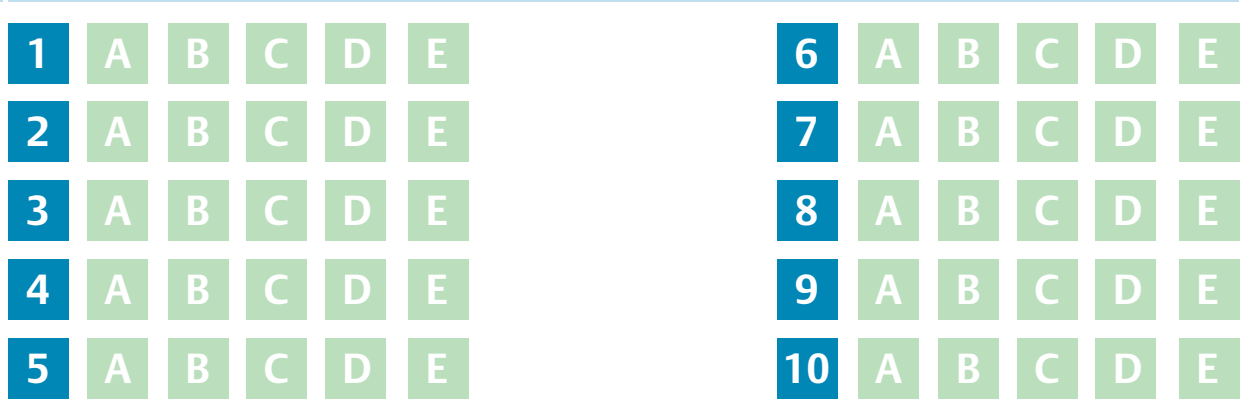

\section{Erklärung}

Ich versichere, dass ich die Beantwortung der Fragen selbst und ohne Hilfe durchgeführt habe

Ort | Datum: $\quad$ Unterschrift:

\section{Feld für CME-Wertmarke}

$>$ Bitte unbedingt Rückseite ausfüllen!

\section{E Zertifizierungsfeld}

Bitte in dieses Feld die CME-Wertmarke kleben oder Ihre Abonnement-Nummer eintragen: (siehe Adressaufkleber) Zertifizierungsfeld (wird durch den Verlag ausgefüllt)

\section{Ihr Ergebnis}

Sie haben von 10 Fragen richtig beantwortet.

Sie haben

bestanden und 3 CME-Punkte erworben.

nicht bestanden

Stuttgart, den

Datum

Stempel/Unterschrift 
F Fragen zur Zertifizierung

Eine Antwort pro Frage. Bitte unbedingt ausfüllen bzw. ankreuzen, da die Evaluation sonst unvollständig ist!

\section{Didaktisch-methodische Evaluation}

1 Das Fortbildungsthema kommt in meiner ärztlichen Tätigkeit

häufig vor

regelmäßig vor

gar nicht vor

2 Das Fortbildungsthema kommt in meiner ärztlichen Tätigkeit

eine feste Gesamtstrategie

noch offene Einzelprobleme:

keine Strategie

3 Hinsichtlich des Fortbildungsthemas

fühle ich mich nach dem Studium des Beitrags in meiner Strategie bestätigt

habe ich meine Strategie verändert:

habe ich erstmals eine einheitliche Strategie erarbeitet

habe ich keine einheitliche Strategie erarbeiten können

4 Wurden aus der Sicht Ihrer täglichen Praxis heraus wichtige Aspekte des Themas nicht erwähnt?

zu knapp behandelt?

\begin{tabular}{|l|l|}
\hline ja welche \\
\hline ja welche \\
\hline ja welche \\
\hline
\end{tabular}

nein

überbewertet

nein

nein

\section{Verständlichkeit des Beitrags}

Der Beitrag ist nur für Spezialisten verständlich

Der Beitrag ist auch für Nicht-Spezialisten verständlich

6 Beantwortung der Fragen

Die Fragen lassen sich aus dem Studium des Beitrages allein beantworten

Die Fragen lassen sich nur unter Zuhilfenahme zusätzlicher Literat beantworten

7 Die Aussagen des Beitrages benötigen eine ausführlichere Darstellung

zusätzlicher Daten

von Befunden bildgebender Verfahren

die Darstellung ist ausreichend

8 Wieviel Zeit haben Sie für das Lesen des Beitrages und die Bearbeitung des Quiz benötigt?

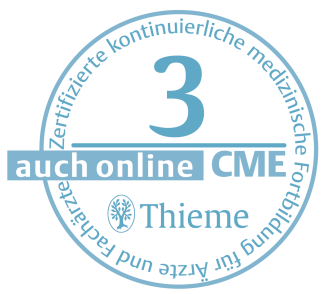

